\title{
Immune response and protective effect against chronic Toxoplasma gondii infection induced by vaccination with a DNA vaccine encoding profilin
}

Qi Gao ${ }^{1,2+}$, Nian-Zhang Zhang ${ }^{1 *+}$, Fu-Kai Zhang ${ }^{1}$, Meng Wang ${ }^{1}$, Ling-Ying Hu ${ }^{1,3}$ and Xing-Quan Zhu $u^{1,4}$

\begin{abstract}
Background: Toxoplasma gondii is an obligate intracellular parasite that can infect almost all warm-blooded animals. T. gondii profilin (TgPF) plays a crucial role in parasite motility and host cell invasion, and has shown promise against toxoplasmosis. DNA vaccine was considered to elicit effective humoral and cell-mediated immunity against T. gondii infection. The objective of the present study was to evaluate the immunogenicity of TgPF in mice using a DNA vaccination strategy.
\end{abstract}

Methods: A DNA vaccine (pVAX-PF) encoding TgPF gene was constructed and then was intramuscularly injected into mice with and without a plasmid encoding IL-15 (pVAX-IL-15). The immune responses in immunized Kunming mice including lymphocyte proliferation, levels of cytokines, antibody titers and T lymphocyte subclasses were analyzed. The protective efficacy against chronic T. gondii infection was observed at 4 weeks post-infection with the cyst-forming PRU strain of T. gondii (Genotype II).

Results: EitherpVAX-PF with or without pVAX-IL-15 could elicit higher level of IgG and IgG2a antibodies and produce strong cellular immune responses in the immunized mice. The brain cyst numbers in mice immunized with pVAX-PF + pVAX-IL-15 (1843 \pm 215.7$)$ and PVAX-PF (1897 \pm 337.8$)$ were reduced $40.82 \%$ and $39.08 \%$, respectively, compared to that in mice received nothing (3114 \pm 168.8$)$, and the differences were statistically significant $(P<0.0001)$. However, the T. gondii cyst numbers in mice immunized with pVAX-PF + pVAX-IL-15 were not statistically significantly different compared to that in mice immunized with pVAX-PF alone [t(10) $=0.33, P>0.05]$.

Conclusions: The present study indicated that TgPF could be a promising vaccine candidate against chronic toxoplasmosis, which can be further used to develop multi-epitope vaccine formulations in food-producing animals against $T$. gondii infection.

Keywords: Toxoplasma gondii, Profilin, Immunogenicity, DNA vaccine, Chronic toxoplasmosis

\section{Background}

The obligated intracellular protozoan Toxoplasma gondii can infect most all of warm-blooded animals and humans, which would lead to zoonotic toxoplasmosis worldwide $[1,2]$. T. gondii usually cause subclinical infection in most

\footnotetext{
* Correspondence: nianzhang919@163.com

${ }^{\dagger}$ Equal contributors

'State Key Laboratory of Veterinary Etiological Biology, Key Laboratory of Veterinary Parasitology of Gansu Province, Lanzhou Veterinary Research Institute, Chinese Academy of Agricultural Sciences, Lanzhou, Gansu Province 730046, People's Republic of China

Full list of author information is available at the end of the article
}

of immunocompetent adults [3, 4], but the parasite would be a severely risk factor for immunodeficient individuals (HIV-infected patients and transplant recipients), children and pregnancy [5-8]. T. gondii is also a common cause of abortion in sheep and goats, leading to serious economic losses $[6,9,10]$. However, no effective treatment was available to eliminate $T$. gondii cysts by now. Immunoprophylaxis against $T$. gondii would be of high priority for the disease control, as previous reviews noted [10-12].

A number of vaccine candidates, including surface antigens (SAG), rhoptry antigens (ROP), microneme antigens 
(MIC), dense granule antigens (GRA) and some other proteins playing important roles in the life cycle of $T$. gondii have been evaluated against $T$. gondii infection. However, no one can completely protect against tissue cysts, usually lower than $80-90 \%$ protection [11]. DNA-based vaccines were considered to elicit effective humoral and cell-mediated immunity against $T$. gondii invasion in animal models, which have been used in many previous studies [11-13].

Following the parasite invasion, host immune response is successively suffered innate acute response and an Agspecific cell-mediated immune response [14]. The invading parasite in mouse model is primarily recognized by Toll-like receptors (TLRs) of DCs, and then triggers the host's TLRs/MyD88 response [15]. TLR11 and 12 are demonstrated as important receptors for $T$. gondii recognition. Activation of TLR11 and 12 can induce potent cytokine responses, and lack of TLR11 and TLR12 genes, mice were showed rapidly succumb to $T$. gondii infection [16-18]. T. gondii profilin (TgPF), one of the ligands of both TLR11 and 12, is essential for the parasite gliding motility, host cell invasion and egress from host cells in mice [17, 19]. TgPF is also shown to be an immunodominant antigen. Immunization of C57BL/6 mice with TgPF encapsulated in oligomannose-coated liposomes induces protective immunity against infection with $T$. gondii tachyzoites (PLK strain) [20].

DNA vaccination can deliver the expressed protein as an endogenous antigen, and has exhibited promise for defense against toxoplasmosis due to the ability of eliciting effective humoral and cellular immune responses in mice [11]. These findings stimulated to hypothesize whether the endogenous TgPF protein could induce effectively protective responses against infection with $T$. gondii tissue cysts, the primary transmission route of $T$. gondii infection for humans [2].

To examine the immunogenicity of the genetic TgPF antigen, we constructed a DNA vaccine encoding TgPF (pVAX-PF), and used a plasmid encoding murine costimulatory molecule IL-15 (pVAX-IL-15) as genetic adjuvant. The pVAX-PF DNA vaccine with or without pVAX-IL-15 were examined for their ability of eliciting immune responses and their protective efficacy against chronic $T$. gondii infection in a murine model.

\section{Methods}

\section{Mice and parasites}

A total of 108 specific-pathogen-free (SPF) grade female Kunming mice aged six to eight weeks were purchased from Lanzhou University Laboratory Animal Center (Lanzhou, China). All mice were handled in strict accordance with good animal practices according to the Animal Ethics Procedures and Guidelines of the People's Republic of China.
Tissue cysts of the low virulent PRU strain of T. gondii (Genotype II) were preserved in State Key Laboratory of Veterinary Etiological Biology, Lanzhou Veterinary Research Institute, Chinese Academy of Agricultural Sciences, Lanzhou, Gansu Province, China. The cysts of the PRU strain were obtained from the brains of orally infected Kunming mice one month after intragastric administration of the cysts.

\section{Expression of TgPF protein in Escherichia coli}

Total RNA was extracted from T. gondii bradyzoites using E.Z.N.A. Total RNA Kit I (Omega, America). The complete open reading frame (ORF) of TgPF gene was amplified by reverse transcription-polymerase chain reaction (RT-PCR) using a pair of specific primers (prfF: $5^{\prime}-\mathrm{C}$ GG GGTACC ATGTCCGACTGGGACCCTGTTGTCA AGG -3', prfR: 5' - CCG GAATTC TTAGTACCCAGA CTGGTGAAGATACTCG - 3'), designed according to the corresponding sequence of the ME49 strain (ToxoDB: TGME49_293690), in which the Kpn I and EcoR I restriction sites were introduced and underlined. The RT-PCR was performed following the instruction of PrimeScript ${ }^{\circ}$ One Step RT-PCR Kit Ver.2 (TaKaRa, China). Briefly, the program was initiated at $50{ }^{\circ} \mathrm{C}$ for $30 \mathrm{~min}$ to synthesize the first strand cDNA, followed by 35 cycles of $94^{\circ} \mathrm{C}$ for $35 \mathrm{~s}$ (denaturation), $65.5^{\circ} \mathrm{C}$ for $45 \mathrm{~s}$ (annealing), $72{ }^{\circ} \mathrm{C}$ for $50 \mathrm{~s}$ (extension) and a final extension of $72^{\circ} \mathrm{C}$ for $10 \mathrm{~min}$.

The obtained DNA was ligated into pET-30a $(+)$, and then transformed into E. coli BL21 (DE3) strain. The positive recombinant plasmids were identified by both restriction enzyme digestion using Kpn I and EcoR I and sequencing (Sangon, China). The recombinant TgPF $(\mathrm{rTgPF})$ protein was expressed at the condition of $0.8 \mathrm{mmol} / \mathrm{L}$ IPTG (Sangon, China), shaking for $8 \mathrm{~h}$ at $37{ }^{\circ} \mathrm{C}$. The rTgPF protein was purified using Ni-NTA His bind resin (Novagen) according to the manufacturer's instructions and were visualized by the sodium dodecyl sulfate-polyacrylamide gel electrophoresis (SDSPAGE).

\section{Construction of the eukaryotic expression plasmids}

The obtained TgPF gene and the eukaryotic expression plasmid pVAX I (Invitrogen, USA) were digested by restriction enzymes, $K p n$ I and $E c o$ R I, respectively. After purification of the two DNA fragments using the Universal DNA Purification Kit (TIANGEN, China), the TgPF gene was linked to pVAX I by T4 DNA ligase (TaKaRa, China). After identification by sequencing, the positive recombinant plasmid pVAX-TgPF was constructed. The pVAX-IL-15 plasmids were preserved in our laboratory as described previously [21], and were identified by sequencing before use (Sangon, China). Both of the two eukaryotic plasmids were transformed into E. coli $\mathrm{DH} 5 \alpha$ and then were purified by anion 
exchange chromatography (EndoFree Plasmid Giga Kit, Qiagen Sciences, MD, USA) following the manufacturer's instructions and stored at $-20{ }^{\circ} \mathrm{C}$ until use. The concentrations of the two plasmids were determined by spectrophotometer at OD260 and OD280.

\section{Expression of pVAX-PF in vitro}

HEK293 cells were transfected with the pVAX-PF or the empty pVAX I (negative control) using lipofectamine ${ }^{\mathrm{Tx}}$ 2000 reagent (Invitrogen) following the manufacturer's instructions. The expression of pVAX-PF plasmid was examined by the indirect immunofluorescence assay (IFA) at $48 \mathrm{~h}$ after transfection. Briefly, the HEK293 cells were fixed with acetone and then perforated with $0.1 \%$ Triton-X-100 in PBS. The cells were incubated with goat polyclonal antibodies against $T$. gondii tachyzoites (1: 50 in PBS) at $37^{\circ} \mathrm{C}$ for $60 \mathrm{~min}$, followed by fluorescein isothiocyanate (FITC)-labeled anti-goat IgG antibodies (Abcam, UK) diluted 1: 2000 in PBS. The specific fluorescence was imaged using a Zeiss Axioplan fluorescence microscope (Carl Zeiss, Germany).

\section{Immunization and challenge}

Mice were inoculated intramuscularly at bilateral quadriceps with $100 \mu \mathrm{g}$ of pVAX-PF + pVAX-IL-15 (group I), $100 \mu \mathrm{g}$ of pVAX-PF (group II), $100 \mu \mathrm{g}$ of pVAX-IL-15 (group III), $100 \mu \mathrm{g}$ of pVAX I (group IV), or sterile phosphate buffered saline (PBS) alone (group V) (each $100 \mu \mathrm{l})$ for three times at two-week interval. Mice which were not inoculated constituted the blank control (group VI). There were 18 mice in each group. Mice from group III, group IV, group V and group VI were treated as controls. Two weeks after the last immunization, splenocytes were aseptically harvested and the red blood cells were removed. All analyses were performed in triplicate.

To measure the tissue cyst burden in mice, 2 weeks after the third vaccination, six mice in per group were inoculated orally with 10 tissue cysts of the PRU strain and the brain cysts were determined at 4 weeks postinfection.

\section{Determination of antibody titers and isotype}

Blood samples were collected from mice in each group at $0,2,4$ and 6 weeks from the tail vein for analysis of specific antibodies. The IgG subclasses (IgG1 and IgG2a) were examined using the sera collected at 6 weeks. The levels of TgPF-specific immunoglobulin G (IgG), IgG1 and IgG2a were measured by ELISA using SBA Clonotyping System-HRP Kit (Southern Biotech CO., LTD, Birmingham, USA) following the manufacture's instruction. Briefly, the purified rTgPF protein $(5 \mu \mathrm{g} / \mathrm{mL})$ was coated on the 96 -well plates over night at $4{ }^{\circ} \mathrm{C}$. After blocking the non-specific sites by $0.5 \%$ BSA in PBS, the wells were incubated with $100 \mu \mathrm{L}$ of mouse serum sample from each group at $37{ }^{\circ} \mathrm{C}$ for $1 \mathrm{~h}$. Then, the horseradish-peroxidase (HRP) conjugated anti-mouse IgG antibodies (1: 2500 dilutions, $100 \mu \mathrm{L}$ ), or antimouse IgG1 or IgG2a (1: 500 dilutions, $100 \mu \mathrm{L}$ ) were added into each well. The absorbance of each well was examined at $450 \mathrm{~nm}$ after incubating with substrate solution ( $\mathrm{pH} 4.0)$ (1.05\% citrate substrate buffer; $1.5 \%$ ABTS; $0.03 \% \mathrm{H}_{2} \mathrm{O}_{2}$ ) in the dark for $20 \mathrm{~min}$.

\section{Lymphocyte proliferation assay by MTs}

The harvested splenocytes from each mouse were cultured in triplicate at a density of $2 \times 10^{5}$ cells per well in complete medium (DMEM medium $+10 \%$ FCS + $100 \mathrm{U} / \mathrm{mL}$ penicillin/streptomycin). The cells from each group were stimulated with $\operatorname{rTgPF}(10 \mu \mathrm{g} / \mathrm{mL})$ and medium alone, respectively, at $37{ }^{\circ} \mathrm{C}$ in a $5 \% \mathrm{CO}_{2}$ incubator. The proliferative activity was measured using MTs method (Promega, USA) after four days. After examining the $\mathrm{OD}_{570}$ value of wells, the stimulation index (SI) was calculated using the formula $\mathrm{OD}_{570 \mathrm{TLA}} / \mathrm{OD}_{570 \mathrm{M}}$.

\section{Flow cytometry analysis}

The concentration of the purified splenocytes were adjusted into $1 \times 10^{5}$ cells $/ \mathrm{mL}$. Then the phycoerythrin (PE)-labeled anti-mouse CD3 (eBioscience) $(5 \mu \mathrm{g} / \mathrm{mL})$, Allophycocyanin (APC)-labeled anti-mouse CD4 (eBioscience) $(5 \mu \mathrm{g} / \mathrm{mL}$ ) and fluorescein isothiocyanate (FITC)-labeled anti-mouse CD8 (eBioscience) $(5 \mu \mathrm{g} / \mathrm{mL})$ antibodies were used to stain the $\mathrm{T}$ cell subclasses $(\mathrm{CD} 4+$ and $\mathrm{CD} 8+)$ for $30 \mathrm{~min}$ at $4{ }^{\circ} \mathrm{C}$. After washing by PBS, the cultures were fixed with FACScan buffer (1\% FCS and $0.1 \%$ Sodium azide in PBS) and $2 \%$ paraformaldehyde. The $\mathrm{T}$ cell subclasses were measured for fluorescence profiles on a FACScan flow cytometer (BD Bio-sciences) and analyzed by SYSTEM II software (Coulter).

\section{Cytokine assays}

To further evaluate the role of the DNA vaccination, the levels of cytokines including IL-2, IL-4, IL-10 and IFN- $\gamma$ was carried out. Briefly, the purified splenocytes of mice from each group were co-cultured with rTgPF as positive control and medium alone as negative control at $24 \mathrm{~h}$ for IL-2 and IL-4, $72 \mathrm{~h}$ for IL-10, and $96 \mathrm{~h}$ for IFN- $\gamma$. The supernatant was evaluated to the concentration of each cytokine using the commercial ELISA kits (Biolegend, USA). $50 \mu \mathrm{L}$ of Assay Buffer A and equal volume of the supernatant of each cell culture were successively added in to microplate well, incubating for $2 \mathrm{~h}$ at room temperature. Then, $100 \mu \mathrm{L}$ of detection solution was added into each well. After washing the plate for 4 times, Avidin-HRP A solution was added and incubated for $30 \mathrm{~min}$, and subsequently the substrate solution $\mathrm{E}$ was added. The reaction was stopped after adding $100 \mu \mathrm{L}$ of stop solution into each well. The 
absorbance was read at $450 \mathrm{~nm}$. The sensitivity limits for the assays were $20 \mathrm{pg} / \mathrm{ml}$ for IFN- $\gamma, 10 \mathrm{pg} / \mathrm{ml}$ for IL- 4 and IL-10, and $50 \mathrm{pg} / \mathrm{ml}$ for IL-2, respectively. The assay was performed in three independent experiments.

\section{Statistical analysis}

All statistical analyses were performed following the procedure of GraphPad Prism 5.0. The differences of the data regarding antibody responses, lymphoproliferation assays, percentages of CD4+ and CD8+ T cells, cytokine production and brain cyst loading were compared by one-way ANOVA. The difference compared in pairs was calculated by the $t$-test. The results in comparisons between groups were considered different if $P<0.05$.

\section{Results}

\section{Sequencing results of the pVAX-PF and pVAX-IL-15}

After identification by restriction enzyme digestion, the positive PVAX-PF plasmids were then confirmed by sequencing. The resulting sequence of pVAX-PF plasmids had $100 \%$ nucleotide sequence identity with the corresponding sequence of the RH strain (GenBank accession No.: AK223678.1). Sequencing of pVAX-IL-15 showed that no base deletion or change was detected after alignment with the corresponding sequence in GenBank (accession no. NM_008357.1).

\section{Expression of pVAX-PF in vitro}

The expression of pVAX-PF was identified by IFA. Cells transfected with the eukaryotic recombinant plasmid pVAX-PF showed specific green fluorescence. However, there was no green dot in the cells transfected with the same amount of pVAX I (Fig. 1).

\section{Evaluation of the humoral immune responses induced by} DNA immunization

The significantly increased anti-TgPF IgG antibodies after the last booster were detected only in the mice immunized with pVAX-PF $[t(3)=9.721, P<0.01]$ or pVAX-PF + pVAX-IL-15 $[t(3)=13.99, P<0.0001]$. Two weeks after the last immunization, the antibody levels in mice immunized with pVAX-PF $(P<0.01)$ and pVAX-PF + pVAX-IL-15 $(P<0.05)$ were significantly increased than those in the control groups (Fig. 2). Mice from group II produced a slightly higher level of IgG antibody after the third vaccination compared to that from group $\mathrm{I}$, but the difference was not significant $(P>0.05)$.

\section{Examination of the types of immune response}

The IgG isotypes were examined to determine whether a Th1 or Th2 response was elicited in the immunized mice by DNA vaccination. As shown in Fig. 3, both IgG1 and IgG2a antibodies in the sera of mice from group I, II and III were significantly increased at 2 weeks after the last immunization, with higher levels of IgG2a to IgG1. These results indicated that pVAX-IL-15, pVAXPF and pVAX-PF + pVAX-IL-15 are able to elicit a Th1 type immune response.

\section{Analysis of cellular immune responses}

As shown in Table 1, splenocytes from mice in Group I $[\mathrm{F}(4,10)=4.531, P<0.01]$ and Group II $[\mathrm{F}(4,10)=7.675$, $P<0.01]$ were significantly increased after stimulating with rTgPF compared with that in mice from the control groups. The difference in SI between Group I and Group II was not statistically significant $[\mathrm{t}(4)=1.823, P>0.05]$.

The $\mathrm{T}$ cell subclasses of mice immunized with various vaccinations were identified by flow cytometry analysis. Percentages of CD3+CD4+ T lymphocytes from Group I $[\mathrm{F}(3,8)=11.28, P<0.01]$, Group II $[\mathrm{F}(3,8)=$ 10.87, $P<0.01]$ and Group III $[\mathrm{F}(3,8)=49.51, P<0.0001]$ were significantly higher than those in mice received pVAX I, PBS or nothing, and were not statistically different among mice in the three control groups. The ratios of CD3+ CD8+ T cells in the spleens of mice vaccinated with pVAX-PF $[\mathrm{F}(4,10)=1.519, \quad P=0.27]$ and $\mathrm{pVAX}-\mathrm{PF}+$
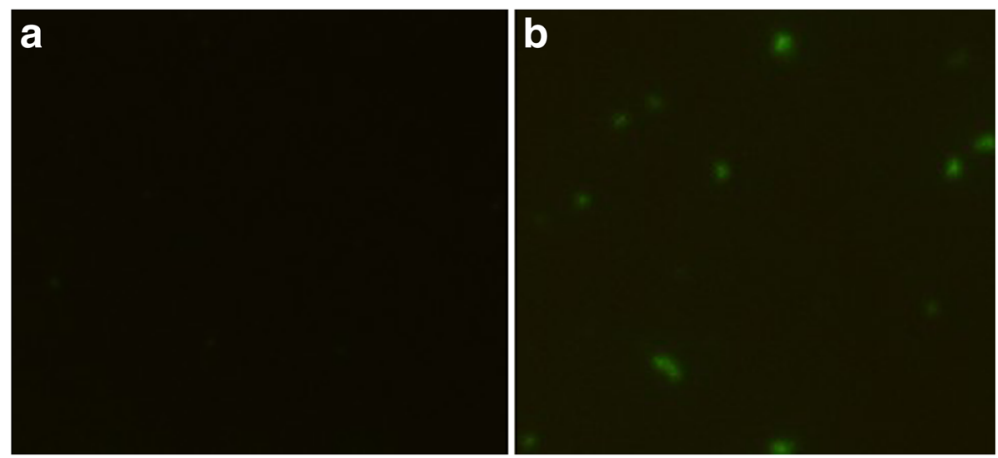

Fig. 1 Analyses of expression of TgPF gene on HEK293 cells by indirect immunofluorescence (IFA) at 48 h post-transfection. a empty vector pVAX l; b HEK293 cells transfected with pVAX-PF 


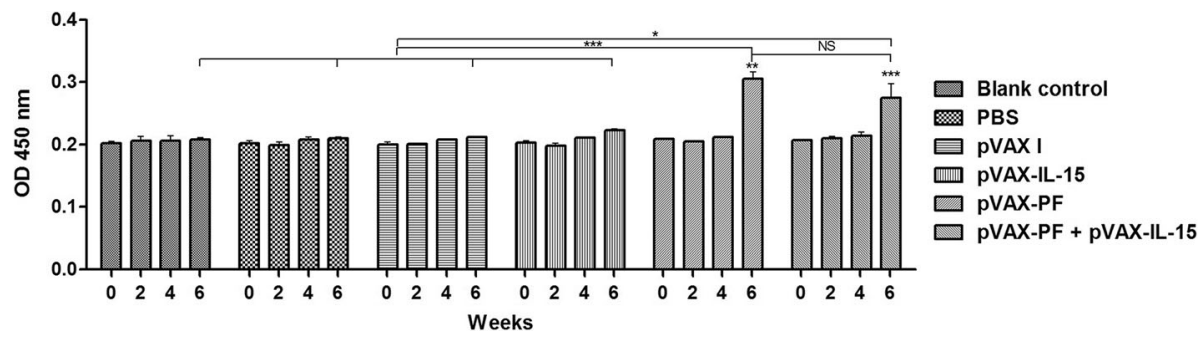

Fig. 2 Determination of IgG antibodies in the sera of Kunming mice at 0,2, 4, and 6 weeks. Mice immunized with pVAX-IL-15, pVAX I, PBS and nothing were treated as controls in statistical analysis. Each bar represents the mean OD $( \pm$ S.E., $n=3) .{ }^{* * *} P<0.0001,{ }^{* *} P<0.01,{ }^{*} P<0.05$, NS: not significant

pVAX-IL-15 $[F(4,10)=0.28, P=0.88]$ was slightly higher than that in all control groups, but the difference was not statistically significant.

\section{Cytokine production by spleen cells}

As shown in Fig. 4, splenocytes in mice from Group I induced significantly higher levels of IL-2 $(P<0.001)$ and IL-10 $(P<0.01)$ compared to that in mice from Group IV, V and VI, but the levels of IFN- $\gamma(P>0.05)$ and IL-4 $(P>0.05)$ were not significantly different. The levels of IFN- $\gamma$, IL-2, IL-4 and IL-10 in spleen cell cultures from pVAX-PF immunized mice were significantly higher than that in the control groups $(P<0.01)$. Mice immunized with pVAX-PF induced significantly higher levels of IFN- $\gamma(P<0.01)$, IL-2 $(P<0.001)$, IL-4 $(P<0.01)$ and IL-10 $(P<0.0001)$ than that in mice immunized with pVAX-PF + pVAX- IL-15.

\section{Assessment of protective activity}

To evaluate whether pVAX-PF with or without pVAX-IL-15 could induce sufficient protection against the formation of $T$. gondii tissue cysts in brain, mice of all the groups were challenged with 10 cysts of $T$. gondii PRU strain and the brain cyst loadings were assessed 28 days after challenge. The number of tissue cysts in mice of Group I $(1843 \pm 215.7)$ and group II $(1897 \pm 337.8)$ were reduced $40.82 \%$ and $39.08 \%$, respectively, compared to that in mice received nothing $(3114 \pm 168.8)$. Immunization with pVAX-PF + pVAX-IL-15 $\quad[\mathrm{F}(4,25)=64.53, \quad P<0.0001]$ and pVAX-PF alone $[F(4,25)=42, P<0.0001]$ significantly decreased the brain cyst formation in mice compared with all the control groups (Fig. 5). The brain cyst numbers in mice from Group I were slightly lower than that from Group II, but the difference was not statistically significant $[\mathrm{t}(10)=0.33, P>0.05]$. Brain cyst loadings in mice immunized with pVAX-IL-15 were significantly lower compared to that in mice vaccinated with pVAX-PF + pVAX-IL-15 $\quad[\mathrm{t}(10)=6.73, \quad P<0.0001]$ and pVAX-PF $[\mathrm{t}(10)=4.30, P<0.01]$, but were significantly higher than that in mice received PVAX I, PBS or nothing $[\mathrm{F}(3,20)=21.11, P<0.0001]$.

\section{Discussion}

In the present study, DNA vaccination of mice with pVAX-PF with or without pVAX-IL-15 could elicit strong humoral and cellular immune responses. The protective activity showed that significant brain cyst reductions were found in mice immunized with pVAXPF and pVAX-PF + pVAX-IL-15 compared to that in

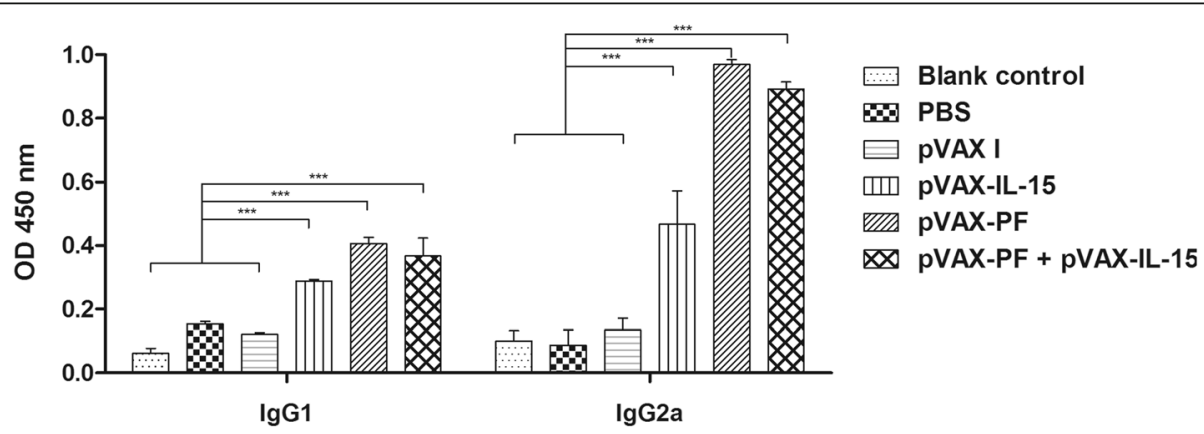

Fig. 3 Analysis of lgG isotypes, IgG1 and lgG2a, in the sera of Kunming mice 2 weeks after the last immunization. Mice immunized with pVAX I, PBS and nothing were treated as controls in statistical analysis. Each bar represents the mean OD $( \pm$ S.E., $n=3)$. ${ }^{* *} P<0.0001$ 
Table 1 Splenocyte proliferative responses and the percentages of T cell subsets in immunized mice 2 weeks after the last immunization

\begin{tabular}{llll}
\hline Groups & SI (Mean \pm SD) & CD3+CD4+ (\%) & CD3+CD8+ (\%) \\
\hline pVAX-PF + pVAX-IL-15 & $1.08 \pm 0.06^{\mathrm{A}}$ & $11.13 \pm 3.02^{\mathrm{A}}$ & $7.13 \pm 1.23^{\mathrm{A}}$ \\
pVAX-PF & $1.13 \pm 0.11^{\mathrm{A}}$ & $11.33 \pm 3.23^{\mathrm{A}}$ & $8.9 \pm 1.13^{\mathrm{A}}$ \\
PVAX-IL-15 & $0.85 \pm 0.05^{\mathrm{B}}$ & $14.53 \pm 1.17^{\mathrm{A}}$ & $6.43 \pm 2.32^{\mathrm{A}}$ \\
pVAXI & $0.79 \pm 0.03^{\mathrm{B}}$ & $3.13 \pm 1.36^{\mathrm{B}}$ & $5.77 \pm 2.6^{\mathrm{A}}$ \\
PBS & $0.85 \pm 0.04^{\mathrm{B}}$ & $3.46 \pm 1.25^{\mathrm{B}}$ & $5.77 \pm 0.67^{\mathrm{A}}$ \\
Blank control & $0.56 \pm 0.01^{\mathrm{C}}$ & $6.43 \pm 1.42^{\mathrm{B}}$ & $6.1 \pm 1.82^{\mathrm{A}}$ \\
\hline
\end{tabular}

Spleens from 3 mice in each group

SI stands for stimulation index

The same superscript letter means no significant difference $(P>0.05)$, whereas different superscript letters mean significant difference $(P<0.05)$

controls, which suggested that the endogenous TgPF protein could strongly induce protective effect against chronic toxoplasmosis. To avoid missing inspection, each sample was examined for 5 times by microscopy. At each time, $10 \mu \mathrm{l}$ of suspensions were dripped onto glass slide, and were checked by three different persons.

IFN $-\gamma$ production and cytotoxic activity of $\mathrm{CD} 8+\mathrm{T}$ cells play important roles for $T$. gondii clearance [22]. The specific CTLs, main subsets of Th1 cells, can directly destroy the host cells containing intracellular niches of T. gondii or through secretion of IFN- $\gamma$ [23]. Another important Th1-skew cytokine for the $T$. gondii resistance is IL-2, which can regulate not only proliferation but also activities of CTLs through an autocrine feedback loop after antigen stimulation [24, 25]. The adaptive immune response is thus induced toward Th1 type $[14,26]$. In the present study, significantly increased levels of IFN- $\gamma$ and IL-2 were detected in mice immunized with pVAX-PF, suggesting induction of a Th1 type immune response, which contributed to the lower brain cyst numbers. Further study should be investigated on the CD4+ and CD8+ T cells excreting cytokines, such as INF- $\gamma$ or IL-12.

Previous studies have shown that IL-15 can prolong the duration of $\mathrm{CD} 8+\mathrm{T}$ cell-mediated immunity [27-29]. Immunizations with IL-15 as adjuvant showed to improve the protective efficacy of vaccine candidates against $T$. gondii infection in Kunming mice [21,30]. Herein, DNA immunization with pVAX-IL-15 can also facilitate specific humoral and cellular immune responses, which further indicated the potential of IL-15 as adjuvant against chronic toxoplasmosis through prolonging the survival of memory T cells [27].

However, the $T$. gondii cyst number in mice immunized with pVAX-PF + pVAX-IL-15 was not significantly different compared to that in mice from Groups II and
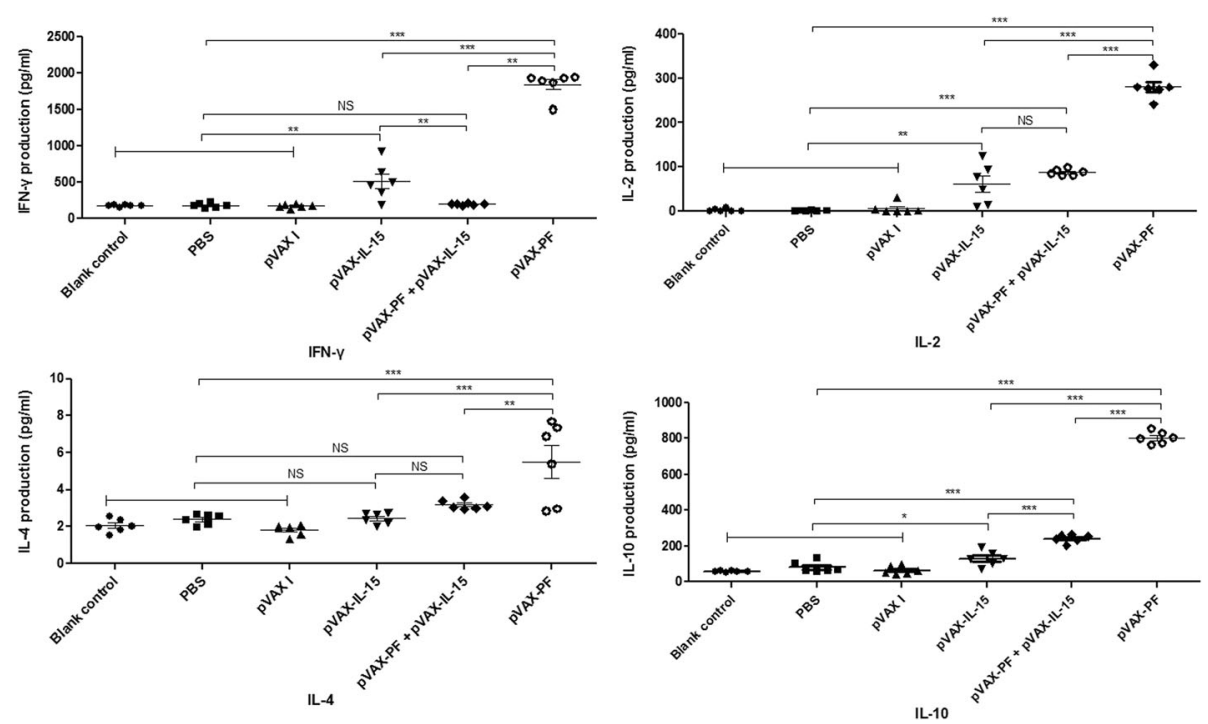

Fig. 4 Cytokine productions secreted by splenocytes of Kunming mice immunized after stimulating by rTgPF protein. Each bar represents the mean OD $( \pm$ S.E., $n=6) .{ }^{* *} P<0.0001,{ }^{* *} P<0.01,{ }^{*} P<0.05$, NS: not significant 


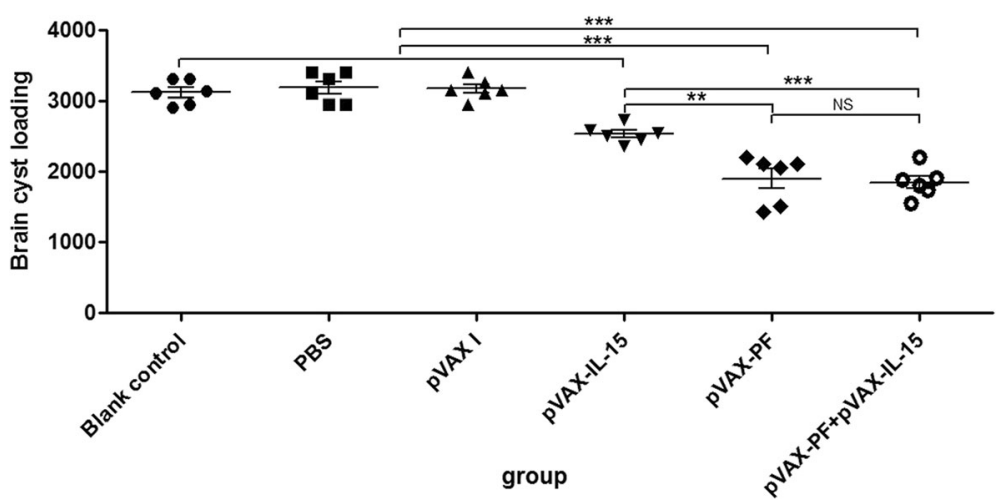

Fig. 5 Protection against chronic toxoplasmosis in mice immunized with pVAX-PF + pVAX-IL-15, pVAX-PF alone and in controls two weeks after the last booster. Mice immunized with pVAX-IL-15, pVAX I, PBS or nothing were treated as controls in statistical analysis. Each bar represents the mean number $( \pm$ S.E., $n=6)$. ${ }^{* * *} P<0.0001 .{ }^{* *} P<0.01$. NS: not significant

III. The humoral and cellular immune responses, such as levels of IgG, IFN- $\gamma$, IL-2 and the percent of CD8+ T cells, in mice immunized with pVAX-PF + pVAX-IL-15 were weak than that in mice immunized with pVAX-PF, which were different to our previous studies using other antigens [21, 30]. IL-15 is structural similar to IL-2, and some subunits of the IL- 15 receptor are shared in common with the receptor of IL-2. Whether the weak effect of co-immunization of pVAX-PF and pVAX-IL-15 is resulting from negative regulation of mutual activities through IL-15 completing the common receptors with IL-2 evoked by pVAX-PF should be further studied.

Specific antibody could attach the parasite to the host cell receptors or the complement molecular. The humoral responses thus are also considered to play an important role in immunity against $T$. gondii [31]. Herein, the specific IgG antibodies were firstly identified by western blotting after the purified rTgPF separated by SDS-PAGE (data not shown). The enhanced humoral immune response including IgG and IgG2a antibodies in mice immunized with pVAX-PF + pVAX-IL-15 or pVAX-PF would be essential for the decrease of brain cyst number. The results were consistent with our previous studies vaccinating mice with plasmids coding CDPK1 [30], ROM4 and ROM5 [32], or immunizing with subunit vaccine [33].

\section{Conclusions}

In conclusion, the present study indicated that immunization with DNA vaccine encoding PVAX-PF with or without pVAX-IL-15 could significantly decrease the brain cyst loadings established in Kunming mice compared to controls, with higher level of IgG and IgG2a antibodies and a strong cellular immune response, which suggested that TgPF would be a good candidate for development of vaccines against $T$. gondii cyst formation used in food-producing animals.

\section{Acknowledgements}

This work was supported, in part, by grants from the National Key Research and Development Program of China (2017YFD0501304), the Elite Program of Chinese Academy of Agricultural Sciences and the Agricultural Science and Technology Innovation Program (ASTIP) (Grant No. CAAS-ASTIP-2016-LVRI03).

\section{Availability of data and materials}

The datasets used and/or analysed during the current study are available from the corresponding author on reasonable request.

\section{Authors' contributions}

XQZ and NZZ conceived and designed the study, and critically revised the manuscript. QG, FKZ, LYH and MW performed the experiments, analyzed the data, and drafted the manuscript. MW and NZZ participated in the study design, study implementation, and manuscript revision. All the authors have read and approved the final manuscript.

\section{Ethics approval and consent to participate}

This study was approved by the Animal Administration and Ethics Committee of Lanzhou Veterinary Research Institute, Chinese Academy of Agricultural Sciences (Approval No. LVRIAEC2013-016).

\section{Consent for publication}

Not applicable

\section{Competing interests}

The authors declare that they have no competing interests.

\section{Publisher's Note}

Springer Nature remains neutral with regard to jurisdictional claims in published maps and institutional affiliations.

\footnotetext{
Author details

${ }^{1}$ State Key Laboratory of Veterinary Etiological Biology, Key Laboratory of Veterinary Parasitology of Gansu Province, Lanzhou Veterinary Research Institute, Chinese Academy of Agricultural Sciences, Lanzhou, Gansu Province 730046, People's Republic of China. ${ }^{2}$ Hunan Entry-exit Inspection and Quarantine Bureau, Changsha, Hunan Province 410004, People's Republic of China. ${ }^{3}$ College of Animal Science, Fujian Agriculture and Forestry University, Fuzhou, Fujian Province 350002, People's Republic of China. ${ }^{4}$ Jiangsu Co-innovation Center for the Prevention and Control of Important Animal Infectious Diseases and Zoonoses, Yangzhou University College of Veterinary Medicine, Yangzhou, Jiangsu Province 225009, People's Republic of China.
} 
Received: 6 April 2017 Accepted: 28 February 2018

Published online: 07 March 2018

\section{References}

1. Dubey JP. Toxoplasmosis of animals and humans. 2nd ed. Boca Raton: CRC Press Inc; 2010. p. 1-313.

2. Robert-gangneux F, Darde ML. Epidemiology of and diagnostic strategies for toxoplasmosis. Clin Microbiol Rev. 2012;25:264-96.

3. Zhou P, Chen Z, Li HL, Zheng H, He S, Lin RQ, et al. Toxoplasma gondi infection in humans in China. Parasite Vectors. 2011;4:165.

4. Dubey JP, Tiao N, Gebreyes WA, Jones JL. A review of toxoplasmosis in humans and animals in Ethiopia. Epidemiol Infect. 2012;140:1935-8.

5. Luft BJ, Remington JS. Toxoplasmic encephalitis in AIDS. Clin Infect Dis. 1992;15:211-22

6. Kravetz JD, Federman DG. Toxoplasmosis in pregnancy. Am J Med. 2005; 118:212-6

7. Weiss LM, Dubey JP. Toxoplasmosis: A history of clinical observations. Int J Parasitol. 2009;39:895-901.

8. Abamecha F, Awel H. Seroprevalence and risk factors of Toxoplasma gondii infection in pregnant women following antenatal care at Mizan Aman general hospital, bench Maji zone (BMZ), Ethiopia. BMC Infect Dis. 2016;16:460.

9. Buxton D. Protozoan infections (Toxoplasma gondii, Neospora caninum and Sarcocystis spp.) in sheep and goats: recent advances. Vet Res. 1998;29:289-310.

10. Lautenslager JP. Toxoplasmosis as a significant disease in man and animals with special reference to preventive measures by the farm community. Can Vet J. 1987:28:261-4

11. Zhang NZ, Chen J, Wang M, Petersen E, Zhu XQ. Vaccines against Toxoplasma gondii: new developments and perspectives. Expert Rev Vaccines. 2013;12:1287-99.

12. Zhang NZ, Wang $M, X u Y$, Petersen $E$, Zhu XQ. Recent advances in developing vaccines against Toxoplasma gondii: an update. Expert Rev Vaccines. 2015;14:1609-21.

13. Han Y, Zhou A, Lu G, Zhao G, Wang L, Guo J, et al. Protection via a ROM4 DNA vaccine and peptide against Toxoplasma gondii in BALB/C mice. BMC Infect Dis. 2017:17:59.

14. Sibley LD. Invasion and intracellular survival by protozoan parasites. Immunol Rev. 2011:240:72-91.

15. LaRosa DF, Stumhofer JS, Gelman AE, Rahman AH, Taylor DK, Hunter CA, Turka LA. T cell expression of MyD88 is required for resistance to Toxoplasma gondii. Proc Natl Acad Sci U S A. 2008;105:3855-60.

16. Koblansky AA, Jankovic D, Oh H, Hieny S, Sungnak W, Mathur R, et al. Recognition of profilin by toll-like receptor 12 is critical for host resistance to Toxoplasma gondii. Immunity. 2013:38:119-30.

17. Yarovinsky F, Zhang D, Andersen JF, Bannenberg GL, Serhan CN, Hayden MS, et al. TLR11 activation of dendritic cells by a protozoan profilin-like protein. Science. 2005;308:1626-9.

18. Yarovinsky F, Hieny S, Sher A. Recognition of Toxoplasma gondii by TLR11 prevents parasite-induced immunopathology. J Immunol. 2008;181:8478-84.

19. Plattner F, Yarovinsky F, Romero S, Didry D, Carlier MF, Sher A, et al. Toxoplasma profilin is essential for host cell invasion and TLR11-dependent induction of an Interleukin-12 response. Cell Host Microbe. 2008;3:77-87.

20. Tanaka S, Kuroda Y, Ihara F, Nishimura M, Hiasa J, Kojima N, et al. Vaccination with profilin encapsulated in oligomannose-coated liposomes induces significant protective immunity against Toxoplasma gondii. Vaccine. 2014;32:1781-5.

21. Li ZY, Chen J, Petersen E, Zhou DH, Huang SY, Song HQ et al. Synergy of mIL-21 and mIL-15 in enhancing DNA vaccine efficacy against acute and chronic Toxoplasma gondii infection in mice. Vaccine. 2014;32:3058-65.

22. Suzuki Y, Orellana MA, Schreiber RD, Remington JS. Interferon-gamma: the major mediator of resistance against Toxoplasma gondii. Science. 1988;240: 516-8.

23. Sturge CR, Benson A, Raetz M, Wilhelm CL, Mirpuri J, Vitetta ES, et al. TLRindependent neutrophil-derived IFN- $\gamma$ is important for host resistance to intracellular pathogens. Proc Natl Acad Sci U S A. 2013;110:10711-6.

24. Boyman O, Sprent J. The role of interleukin-2 during homeostasis and activation of the immune system. Nat Rev Immunol. 2012;12:180-90.

25. Sa Q, Jerold W, Yasuhiro S. IL-2 produced by CD8+ immune T cells can augment their IFN- $\gamma$ production independently from their proliferation in the secondary response to an intracellular pathogen. J Immunol. 2013;190: 2199-207.
26. Suzuki $Y$, Remington JS. The effect of anti-IFN-g antibody on the protective effect of Lyt-21 immune T cells against toxoplasmosis in mice. J Immunol. 1990;144:1954-6.

27. Khan IA, Casciotti L. IL-15 prolongs the duration of CD8+ T cell-mediated immunity in mice infected with a vaccine strain of Toxoplasma gondii. J Immunol. 1999;163:4503-9.

28. Khan IA, Kasper LH. IL-15 augments CD8+ T cell-mediated immunity against Toxoplasma gondii infection in mice. J Immunol. 1996;157:2103-8.

29. Bhadra R, Guan H, Khan IA. Absence of both IL-7 and IL-15 severely impairs the development of CD8 T cell response against Toxoplasma gondii. PLOS One. 2010:5:e10842.

30. Chen J, Li ZY, Huang SY, Petersen E, Song HQ, Zhou DH, et al. Protective efficacy of Toxoplasma gondii calcium-dependent protein kinase 1 (TgCDPK1) adjuvated with recombinant IL-15 and IL-21 against experimental toxoplasmosis in mice. BMC Infect Dis. 2014;14:487.

31. Kang H, Remington JS, Suzuki Y. Decreased resistance of B cell deficient mice to infection with $T$. gondii despite unimpaired expression of IFNgamma, TNF-alpha and inducible nitric oxide synthase. J Immunol. 2000; 164:2629-34.

32. Zhang NZ, Xu Y, Wang M, Petersen E, Chen J, Huang SY, et al. Protective efficacy of two novel DNA vaccines expressing Toxoplasma gondii rhomboid 4 and rhomboid 5 proteins against acute and chronic toxoplasmosis in mice. Expert Rev Vaccine. 2015;14:1289-97.

33. Zhang NZ, Xu Y, Wang M, Chen J, Huang SY, Zhu XQ. Vaccination with Toxoplasma gondii calcium-dependent protein kinase 6 and rhoptry protein 18 encapsulated in poly(lactide-co-glycolide) microspheres induces longterm protective immunity in mice. BMC Infect Dis. 2016;16:168.

\section{Submit your next manuscript to BioMed Central and we will help you at every step:}

- We accept pre-submission inquiries

- Our selector tool helps you to find the most relevant journal

- We provide round the clock customer support

- Convenient online submission

- Thorough peer review

- Inclusion in PubMed and all major indexing services

- Maximum visibility for your research

Submit your manuscript at www.biomedcentral.com/submit
) Biomed Central 